\title{
Use of High-Power Combiners and Fast Directional Switches in ECRH Systems
}

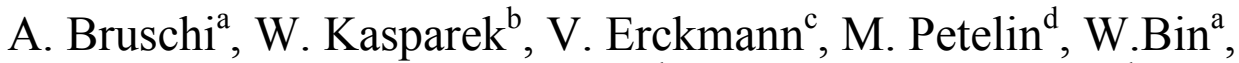 \\ O.D'Arcangelo ${ }^{\mathrm{a}}$, L. Lubyako ${ }^{\mathrm{d}}$, V. Muzzini ${ }^{\mathrm{a}}$, B. Plaum ${ }^{\mathrm{b}}$. \\ ${ }^{a}$ Istituto di Fisica del Plasma, CNR, Milano, Italy \\ ${ }^{b}$ Institut für Plasmaforschung, Universität Stuttgart, Germany \\ ${ }^{c}$ Max-Planck-Institut für Plasmaphysik, Greifswald, Germany \\ ${ }^{d}$ Institute of Applied Physics, RAS, Nizhny Novgorod, Russia
}

\begin{abstract}
The new generation of compact devices for the combination and the fast switching of high-power millimeter-wave beams [1] for Electron Cyclotron Heating (ECH) gives the possibility to switch the power (in tens of microseconds) between two lines (or two ECH launchers, even modulating it between them) and combine two gyrotron sources (or in principle even more) in one single transmission line, for doubling the transmitted power. This is useful in many respects in order to: 1) double the efficiency in modulated EC for neoclassical Tearing Modes (NTM) stabilization, 2) avoid to switch-off gyrotrons in conventional (slow) switching, 3) electronically control the power sharing between different applications (heating/current-drive or NTM stabilization), 4) upgrade the existing ECH systems to twice the power without adding complete transmission lines and launchers, 5) test components at a power doubled with respect to the power capability of the available sources. This opens the way to the development of a more effective "active" real-time control of the ECRH power routing and generally to more flexible and powerful ECH systems. The development of different devices and the advantages for (and in view of) ITER are addressed.
\end{abstract}

Keywords: beam switches, mm-wave combiners, electron cyclotron heating.

PACS: 42.60.Da, 52.50.Sw, 84.40.-x

\section{INTRODUCTION}

The development of present Electron Cyclotron Heating (ECH) experiments includes the possibility of using ECH in plasmas for a number of different tasks. Among the others, besides Neo-classical Tearing Modes (NTM) stabilization and sawtooth control already envisaged for ITER, presently also startup/ramp-up assistance and disruption avoidance are under study. In the design of the ECH systems, however, the flexibility to use routinely ECH as a tool for plasma control is not implemented. Present-day transmission lines connect directly sources to launchers without redundancy and only mechanical switches (inserted in the optical path or in the waveguide) can be used for routing the power. In case of ITER, the ECH system will launch at least $20 \mathrm{MW}$ of EC power coming from 24 Gyrotrons $(f=170 \mathrm{GHz}, 1-2 \mathrm{MW})$ in four Upper ports (dedicated to NTM stabilization and sawtooth control) or alternatively in one Equatorial port, dedicated to heating and current-drive. If ECH is used for sustainment 
of a standard scenario where NTM could destabilize, in case they do, all or part of the power must be switched to the dedicated Upper launchers. The number of the gyrotrons needed, the timing of their effective use, the speed at which they intervene is important to limit the growth rate and the development of the mode, since early stabilization may require less power. The way to accomplish this task foresees switching-off some (all?) of the gyrotrons, turning the mechanical switches to the desired line and switching the gyrotrons on again, with a delay of the order of seconds (Fig.1). This has a strong effect also on the base scenario, since power requested is no more usable for scenario sustainment. Such a delay from mode detection and decision (to switch to another launcher) to the effective time of intervention on the mode (reduction or stabilization) is an obstacle to efficient use of the power for mode control. In fact how many gyrotron to use (switch) initially and how many to keep on the mode location during the discharge has to be decided a few seconds before they can be used or released. This means that a (big) margin should be considered on the power dedicated to NTM stabilization "ab initio", or the risk of island growth and subsequent large deployment of sources must be taken. Moreover the reduction of heating power modifies plasma conditions and the mode itself. The control system in such a situation can work with reaction time of the order of seconds, with danger of oscillating behaviour (with sources switched between heating and stabilization purposes) unless an adequate number of sources are dedicated steadily to stabilization purposes and not to heating.

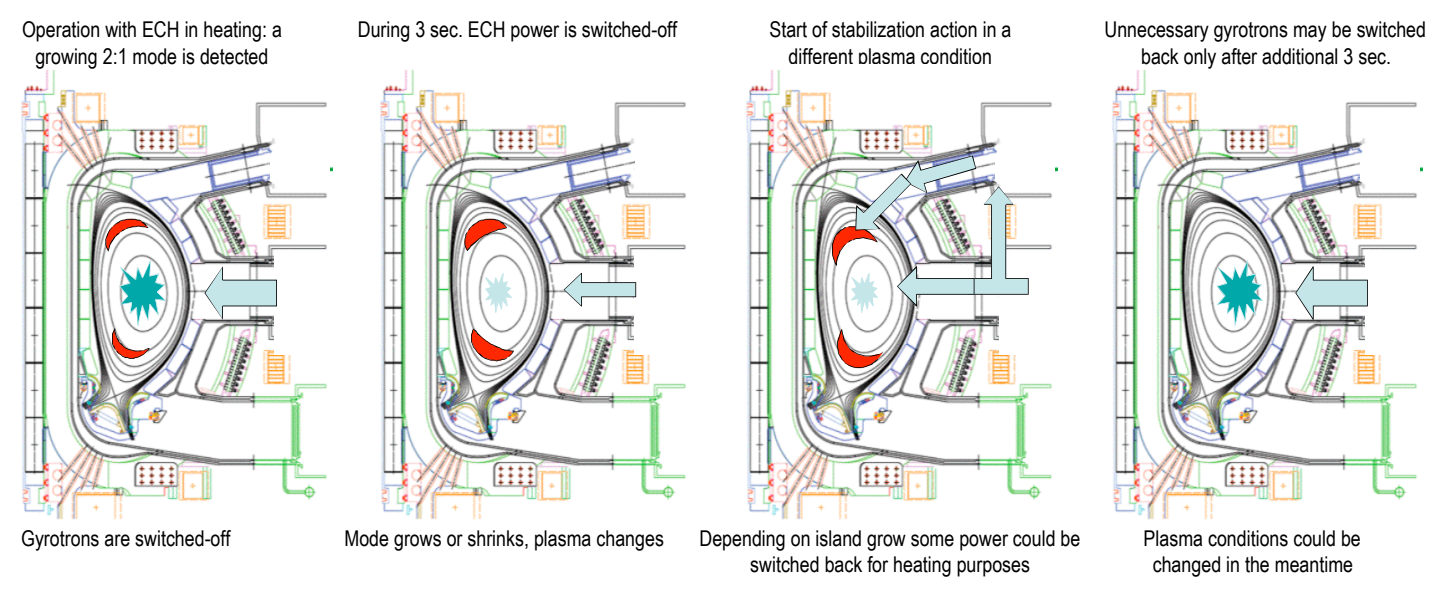

FIGURE 1. From left to right: possible steps for gyrotron use during EC heating: from the detection of the mode (left) to the start of the stabilization action, during a few second of reduced power due to gyrotron inactivity the plasma condition may change: subsequent stabilization may require less power, allowing sources to be re-directed to heating purposes, again after a few seconds of inactivity.

\section{FAST DIRECTIONAL SWITCHES}

Now, new fast directional switches (FADIS) are becoming available for reducing the switching time down to tens of microseconds, preventing also the troubles of switching-off and on the gyrotrons. The devices are presently in different stage of development: the most advanced are being tested at high power in these days. They are essentially passive devices that use constructive/destructive interference of a beam 
inserted at one input and divided in parts in such a way to add or cancel the power at one or the other output, depending on the phase difference (Fig.2). Switching is realized with a change of phase of $\pi$ on the characteristic length $L$ of the device, path difference for interferometers or loop length for resonators, typically a few meters. The power splitting ratio may be changed continuously between the two outputs, without the need for switching off the source either with a change of the source frequency by $\Delta \omega / \omega \sim \lambda / 2 \mathrm{~L}$ (tens of $\mathrm{MHz}$ for hundred $\mathrm{GHz}$, realized in tens of microseconds with a change or modulation of the beam voltage between two levels) or of the length L (obtained in milliseconds, with mechanical tuning of a mirror position).

Switch

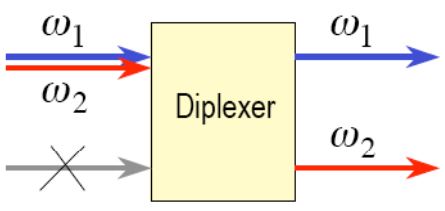

Combine and Switch

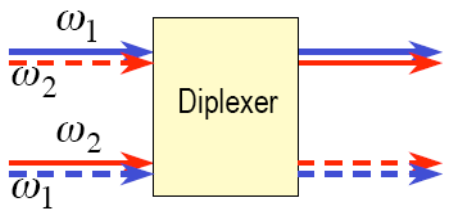

FIGURE 2. Schematic view of the device use as fast switch (left) and as switching-combiner (right).

Many are the design options, mainly dependent on the splitting and transmitting media (see Fig.3). Among splitting media diffraction gratings [1], dielectric sheets and square corrugated waveguides ( $\mathrm{SCW}$ ) exploiting the multiple imaging due to the Talbot Effect [2]. Among the realized devices, some were tested at low power, some other already demonstrated the high-power capability [3]. A new version of grating resonator thought for application on ASDEX-U is presently under test on the ECH system of W7-X. A plan for testing a SCW on the FTU ECRH plant is under discussion. Application of the new devices in present ECRH systems aims at the demonstration of power handling capability, compatibility to the application on real plasmas and to effective use in feedback-controlled experiments.

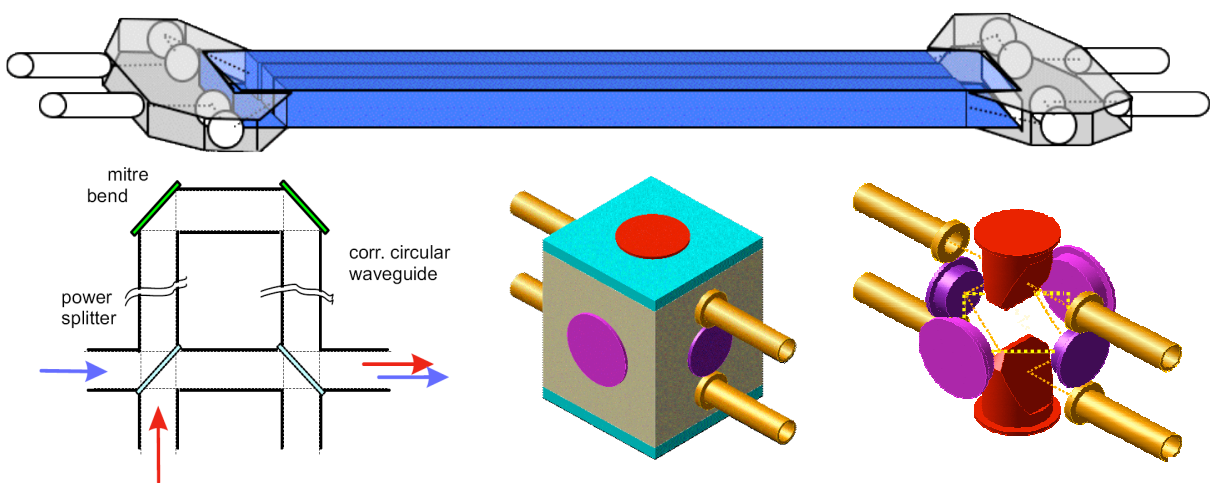

FIGURE 3. Versions of diplexer/combiners: double-loop SCW resonator (top), in-waveguide dielectric-splitter interferometer (left), grating resonator (center, right)

\section{USE OF FADIS FOR ITER}

The device can be exploited, for its fast response and electronic drive, in ECH transmission lines (in ITER and also in all multi-gyrotron ECH systems) in order to 
control the power sharing among different applications (heating or stabilization), for increasing system efficiency, for upgrading existing systems to double power using the same transmission lines and launchers. A possible upgrade for ITER ECH, could be the replacement of the mechanical switches directing the power to the equatorial or upper launchers with fast ones (see Fig.4). In a first step this would allow to control the power nearly instantaneously between the launchers, additionally leaving, in case of modulated NTM stabilization, half of the power in the equatorial. In a second step an upgrade of the power would require only new sources and transmission lines up to the FADIS, without doubling the system, with the possibility to use all the power available both for heating and stabilization. A third step (or an alternative step for increasing the power of the system) could be the use of dedicated combiners at the source side. Combination would also allow testing of ECH components at $2 \mathrm{MW}$, since no source still exists [4].

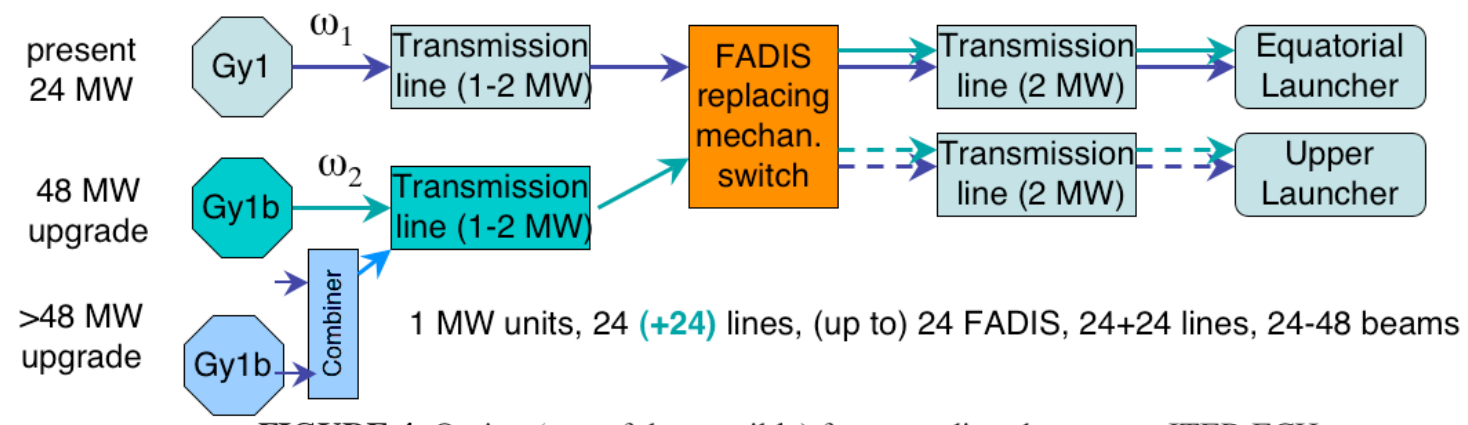

FIGURE 4. Option (one of the possible) for upgrading the present ITER ECH system

\section{CONCLUSIONS}

The demonstration of $1 \mathrm{MW} \mathrm{CW}$ switching and combination capability is under way. In parallel, techniques for the control of gyrotron frequency with minimum reduction of the power are being studied. The feasibility of the application in different environments (evacuated waveguides, quasi-optical transmission lines) is guaranteed by the wide spectrum of characteristics (size, shape...) of the versions available.

\section{ACKNOWLEDGMENTS}

This work is carried out in the frame of the Virtual Institute "Advanced ECRH for ITER" (collaboration between IPP Garching and Greifswald, FZK Karlsruhe, IHE University of Karlsruhe, IPF Stuttgart, IAP Nizhny Novgorod and IFP Milano), supported by the Helmholtz-Gemeinschaft deutscher Forschungszentren.

\section{REFERENCES}

1. W. Kasparek et al., Nucl. Fusion 48 (2008) 054010.

2. A.Bruschi et al.,, Fusion Sci. Technol. 53 (2008), 97-103.

3. W. Kasparek et al., 5th IAEA-TM on ECRH Physics and Technology, Gandhinagar, India, Feb 2009.

4. V.Erckmann et al., 5th IAEA-TM on ECRH Physics and Technology, Gandhinagar, India, Feb 2009. 